\title{
Intracranial hypertension and HIV associated meningoradiculitis
}

\author{
M C Prevett, G T Plant
}

\begin{abstract}
Two patients with meningoradiculitis associated with HIV presented with symptoms and signs of intracranial hypertension. In the patients described, the raised intracranial pressure resolved after lumbar puncture. After exclusion of opportunistic infection, such patients may be managed with therapeutic lumbar puncture alone.
\end{abstract}

$(\mathcal{F}$ Neurol Neurosurg Psychiatry 1997;62:407-409)

Keywords: Intracranial hypertension; HIV seropositivity

Opportunistic infection and neoplasms of the nervous system are common complications of AIDS. In addition, HIV is neurotropic, and neurological involvement may occur at any stage during the course of HIV infection from the time of seroconversion. ${ }^{1}$ In this report we describe two patients who presented with intracranial hypertension early in the course of HIV infection.

\section{Patient 1}

A 41 year old African woman presented with a nine month history of headache and one week of visual obscurations and tinnitus. She had lost $17 \mathrm{~kg}$ in weight in the preceding year, but there were no other systemic symptoms, and she was not on any medication. She had visual acuities of $6 / 5$ bilaterally with normal colour vision. Perimetry showed symmetric enlargement of the blind spots and, on fundoscopy, bilateral chronic papilloedema was seen. Neurological and general examinations were otherwise normal.

Brain MRI and magnetic resonance angiography (MRA) were normal. At lumbar puncture, CSF pressure was raised at $38.5 \mathrm{~cm}$ water with a protein concentration of $0.77 \mathrm{~g} / 1$ and 27 white cells $/ \mathrm{mm}^{3}$ (97\% lymphocytes). Glucose concentration in CSF was $3.2 \mathrm{mmol} / 1$ (blood glucose $5.4 \mathrm{mmol} / \mathrm{l}$ ). Oligoclonal bands were detected in CSF and serum, but there were additional bands in the CSF not present in the serum. Culture of CSF was negative for bacteria, fungi, and acid fast bacilli; CSF cryptococcal antigen, polymerase chain reaction for toxoplasmosis, and syphilis serology were negative. Antibodies to HIV-1 and HIV-2 were detected in serum. Cytomegalovirus serology was positive (IgG positive, IgM negative), but CSF and other viral serology were negative. There was a mild normochromic, normocytic anaemia (haemoglobin $11 \cdot 2 \mathrm{~g} / \mathrm{dl}$ ) and a lymphopenia $\left(1 \cdot 1 \times 10^{9} / 1\right)$ with a CD4 count of $260 \times 10^{6} / 1$. Erythrocyte sedimentation rate was $61 \mathrm{~mm} / \mathrm{h}$, and total serum protein was raised at $93 \mathrm{~g} / 1$ due to a polyclonal increase in $\gamma$ globulins. Routine biochemistry, serum and CSF angiotensin converting enzyme, hepatitis B serology, HTLV 1 serology, clotting screen, autoantibody screen, and chest radiography were normal.

Empirical antituberculous therapy was commenced pending the CSF cultures, but the patient did not comply with the treatment. After the lumbar puncture the headache and papilloedema resolved spontaneously over a period of four weeks, but examination showed loss of the knee and ankle tendon reflexes, without any weakness or sensory disturbance. The upper limb reflexes were normal, and the plantar responses were flexor. Nerve conduction studies were normal. Repeat lumbar puncture disclosed a normal opening pressure of $12.5 \mathrm{~cm}$ water, with a protein concentration of $1 \cdot 27 \mathrm{~g} / 1$ and 34 white cells $/ \mathrm{mm}^{3}(100 \%$ lymphocytes). Three months later, when last assessed, she was complaining of headache again. The papilloedema had not recurred, but the knee and ankle tendon reflexes were still absent. Brain CT was normal, and CSF pressure was $13.5 \mathrm{~cm}$ water. The CSF protein remained increased at $1.13 \mathrm{~g} / \mathrm{l}$, but the white cell count had fallen to 5 cells $/ \mathrm{mm}^{3}$. The headache settled without treatment.

\section{Patient 2}

A 23 year old African woman presented with headache and diplopia. She had been well until seven weeks before presentation when she developed severe intermittent headache. After five weeks of headaches, she developed horizontal diplopia and progressive unsteadiness of gait. There was no medical history of note, and she was not on any regular medication. She had visual acuities of $6 / 6$ bilaterally with normal colour vision. On perimetry, bilateral enlargement of the blind spots was 
found, and fundoscopy disclosed bilateral acute papilloedema with peripapillary haemorrhages, cotton wool spots, and retinal folds. There was bilateral lateral rectus weakness and lower motor neuron facial weakness. Tone and power were normal in the upper limbs, but there was grade 4 weakness of hip flexion, knee flexion, ankle dorsiflexion, and ankle plantarflexion bilaterally. Upper limb reflexes were normal, but the knee and ankle tendon reflexes were absent. Both plantar responses were flexor. Sensation and coordination were normal. There was cervical lymphadenopathy, but general examination was otherwise normal.

Brain MRI and MRA were normal. Spinal MRI showed faint enhancement of the lumbar nerve roots after gadolinium but no other abnormality. Lumbar puncture disclosed a CSF pressure of $39 \mathrm{~cm}$ water with a protein concentration of $1.3 \mathrm{~g} / 1$ and 42 white cells $/ \mathrm{mm}^{3}$ ( $100 \%$ lymphocytes). Oligoclonal bands were present in both CSF and serum. Glucose in CSF was $3.0 \mathrm{mmol} / 1$ (blood glucose $4.6 \mathrm{mmol} / \mathrm{l})$. Culture for bacteria, fungi, and acid fast bacilli was negative. Cryptococcal antigen was not detected in CSF. Nerve conduction studies and EMG were normal. Antibodies to HIV-1 were present in serum. Angiotensin converting enzyme was slightly raised in serum at $56 \mathrm{IU} / 1$ (normal $<53 \mathrm{IU} / \mathrm{ml}$ ), but was normal in CSF. Chest radiography was normal. There was no evidence of granuloma formation or lymphoma on cervical lymph node biopsy or bone marrow biopsy, and culture of both specimens for acid fast bacilli was negative. Full blood count was normal, and the CD4 count was $780 \times$ $10^{6} / 1$. Erythrocyte sedimentation rate was 37 $\mathrm{mm} / \mathrm{h}$, and there was a polyclonal increase in $\gamma$-globulins. Toxoplasma and CMV IgG antibodies (IgM negative) were detected in serum but not CSF; $\mathrm{HTLV}_{1}$, syphilis, and borrelia serology were negative. Routine biochemistry and autoantibody screen were normal.

While awaiting the results of tuberculosis cultures, empirical treatment with rifampicin, isoniazid, pyrazinamide, and ethambutol was started. Subsequently prednisolone $(60 \mathrm{mg} /$ day) was started. The headache, lateral rectus palsies, and facial weakness rapidly resolved after the first lumbar puncture. A lumbar puncture performed one week after presentation disclosed a CSF pressure of $15 \mathrm{~cm}$ water with a protein concentration of $1 \cdot 1 \mathrm{~g} / 1$ and 28 white cells $/ \mathrm{mm}^{3}$. During the first two weeks the power in her lower limbs deteriorated. At three weeks the CSF pressure was $23 \mathrm{~cm}$ water with a protein concentration of $1.2 \mathrm{~g} / 1$ and 5 white cells $/ \mathrm{mm}^{3}$. Repeat nerve conduction studies and EMG, six weeks after the initial examination, were compatible with a lumbosacral radiculopathy with absent peroneal $\mathrm{F}$ waves bilaterally, prolonged $\mathrm{F}$ wave latencies from both tibial nerves, and evidence of denervation in the tibialis anterior and vastus medialis. Motor nerve conduction velocities and sural sensory action potentials remained within normal limits. Two to three months after the initial presentation the power in her lower limbs had almost completely recovered. During the same period the papilloedema resolved. Antituberculous therapy was stopped and a reducing regimen of steroids continued.

\section{Discussion}

Both patients presented with symptoms and signs of raised intracranial pressure without an intracranial mass or ventricular enlargement and were subsequently found to have serological evidence of HIV infection.

Intracranial hypertension is a recognised complication of cryptococcal meningitis in AIDS. ${ }^{1}$ Both our patients had a lymphocytic meningitis, but there was no evidence of cryptococcal infection, and the CD4 counts of greater than 200 make opportunistic infection unlikely. Having made a careful search for other potential causes of chronic meningitis, the lymphocytic meningitis is most likely to have represented an HIV associated meningitis. Up to $60 \%$ of seropositive patients have a chronic low grade meningitis, which is usually asymptomatic. ${ }^{1}$ Intracranial hypertension can occur as a complication of viral meningitis, but it is rare. ${ }^{2}$ In a study of 14 patients with HIV associated meningitis, although the CSF pressure was $20 \mathrm{~cm}$ water or greater in five patients, it was only above $25 \mathrm{~cm}$ in one patient and papilloedema was not seen. ${ }^{3}$

Patient 2 had clinical and neurophysiological evidence of a lumbosacral radiculopathy. This progressed during the first two weeks and then improved over the subsequent two to three months. Bilateral facial weakness was also present, but this resolved rapidly after the initial lumbar puncture suggesting that it was the result of raised intracranial pressure. Patient 1 developed absent knee and ankle tendon reflexes, but there was no weakness. Nerve conduction studies, performed acutely, were normal, but a follow up study was not available. Acute and chronic inflammatory demyelinating neuropathies are rare but well recognised in early HIV infection. ${ }^{4}$ Although the pathogenesis of these neuropathies remains speculative, it has been suggested that autoimmune mechanisms, analogous to those in Guillain-Barré syndrome and chronic inflammatory demyelinating neuropathy unrelated to HIV infection, are involved. ${ }^{4}$ Intracranial hypertension is a recognised complication of both diseases, unrelated to HIV infection, and there is one report of intracranial hypertension developing four weeks after the onset of Guillain-Barré syndrome associated with HIV infection. ${ }^{5}$ The clinical presentation in patient 2 and possibly also patient 1 could be considered similar to those patients reported with Guillain-Barré syndrome and intracranial hypertension.

Four other patients with intracranial hypertension without an intracranial mass or ventricular enlargement have been reported in patients with HIV infection. ${ }^{6-9}$ Unlike our patients, all four were known to be HIV positive when they developed intracranial hypertension and none had a radiculopathy. One patient had neither evidence of opportunistic 
infection nor HIV associated meningitis, ${ }^{7}$ and may have represented a chance occurrence of idiopathic intracranial hypertension and HIV infection. Two patients had an aseptic meningitis, ${ }^{89}$ but both had been receiving co-trimoxazole, which has been previously associated with intracranial hypertension. ${ }^{10}$ Although in one patient the intracranial hypertension recurred after withdrawal of the co-trimoxazole, it is difficult to exclude co-trimoxazole as an aetiological factor in these two patients. In the remaining case, the intracranial hypertension was attributed to treatment with amphotericin. ${ }^{6}$

Although the temporal link with the meningoradiculitis in our patients makes a chance occurrence of idiopathic intracranial hypertension and HIV infection unlikely, and they were not receiving any medication, the mechanism of the raised intracranial pressure is uncertain. Impaired absorptive function of the arachnoid villi secondary to the raised CSF protein concentrations was proposed as an explanation of the raised intracranial pressure in Guillain-Barré syndrome, but this hypothesis has since been questioned. ${ }^{11}$ The most parsimonious explanation is that the chronic meningitis itself caused impaired CSF absorption, and certainly the raised intracranial pressure did not recur when the CSF lymphocytosis had resolved despite persistent raised CSF protein.

In patient 1 , the CSF lymphocytosis and raised intracranial pressure resolved with no treatment other than a lumbar puncture. Patient 2 followed an essentially similar course, and it remains uncertain whether this was influenced by the treatment given. It would seem reasonable, after exclusion of any opportunistic infection, to manage such patients with therapeutic lumbar puncture only.

In summary, two patients presented with intracranial hypertension and an HIV associated meningoradiculitis. The differential diagnosis of intracranial hypertension without an intracranial mass lesion or ventricular enlargement in patients with HIV infection should include idiopathic intracranial hypertension, drug treatment, and cryptococcal meningitis.

1 Harrison MJG, McArthur JC. AIDS and neurology. London: Churchill Livingstone, 1995.

2 Silverstein A. Papilledema with acute viral infections of the brain. Mount Sinai f Med 1974;41:435-43.

3 Hollander $\mathrm{H}$, Stringari S. Human immunodeficiency virusassociated meningitis. Am $₹$ Med 1987;83:813-6.

4 Cornblath DR, McArthur JC, Kennedy PGE, Witte AS, Griffen JW. Inflammatory demyelinating neuropathies associated with human T-cell lymphotropic virus type III infection. Ann Neurol 1987;21:32-40.

5 Gross FJ, Mindel JS. Pseudotumor cerebri and GuillainBarré syndrome associated with human immunodeficiency virus infection. Neurology 1991;41:1845-6.

6 Heudier P, Chichmanian RM, Taillan B, Gratecos N, Fuzibet JG, Dujardin P. Drug-induced benign intracranial hypertension. Apropos of a case with amphotericin B. Review of the literature. Therapie 1992;47:403-7.

7 Traverso F, Stagnaro R, Fazio B. Benign intracrania hypertension associated with HIV infection. Eur Neurol 1993;33:191-2.

8 Javeed N, Shaikh J, Jayaram S. Recurrent pseudotumour cerebri in an HIV-positive patient. AIDS 1995;9:817-9.

9 Schwarz S, Husstedt IW, Georgiadis D, Reichelt D, Zidek W. Benign intracranial hypertension in an HIV-infected patient: headache as the only presenting sign. AIDS patient: headach

10 Ch'ien LT. Intracranial hypertension and sulfamethoxazole. $N$ Engl $₹ \mathrm{Med}$ 1970;283:47.

11 Morley JB, Reynolds EH. Papilloedema and the LandryGuillain-Barré syndrome. Case reports and review. Brain 1966;89:205-22. 